\title{
Predictors of Impaired Postpartum Renal Function in Women after Preeclampsia: Results of a Prospective Single Center Study
}

\author{
T. Kaleta, ${ }^{1}$ A. Stock, ${ }^{1}$ D. Panayotopoulos, ${ }^{2}$ O. Vonend, ${ }^{3}$ D. Niederacher, ${ }^{1}$ \\ M. Neumann, ${ }^{1}$ T. Fehm, ${ }^{1}$ W. Kaisers, ${ }^{4}$ and M. Fleisch ${ }^{5}$ \\ ${ }^{1}$ Department of Obstetrics and Gynaecology, Heinrich-Heine-University Medical Centre, Moorenstrasse 5, \\ 40225 Duesseldorf, Germany \\ ${ }^{2}$ Department of Obstetrics and Gynecology, Dormagen Hospital, Dr.-Geldmacher-Straße 20, 41540 Dormagen, Germany \\ ${ }^{3}$ Department of Nephrology, DKD Helios Clinic Wiesbaden GbR, Aukammallee 33, 65191 Wiesbaden, Germany \\ ${ }^{4}$ Department of Mathematics, Faculty of Mathematics and Natural Sciences, Heinrich-Heine-University, Universitätsstrasse 1, \\ 40225 Düsseldorf, Germany \\ ${ }^{5}$ Department of Obstetrics and Gynaecology, Helios University Medical Center Wuppertal, Heusnerstraße 40, \\ 42283 Wuppertal, Germany
}

Correspondence should be addressed to T. Kaleta; thomas.kaleta@med.uni-duesseldorf.de

Received 29 January 2016; Revised 15 May 2016; Accepted 7 June 2016

Academic Editor: Michael Hawkes

Copyright (C) 2016 T. Kaleta et al. This is an open access article distributed under the Creative Commons Attribution License, which permits unrestricted use, distribution, and reproduction in any medium, provided the original work is properly cited.

Objective. The purpose of this prospective study was to investigate the predictive value of single prepartum findings combined with serum biomarkers sFlt-1 (soluble fms-like tyrosine kinase-1) and PlGF (placental growth factor) indicating severity of preeclampsia (PE) for occurrence and extent of impaired postpartum kidney function. Study Design. In this prospective, single center study $44 \mathrm{PE}$ patients were compared to 39 healthy controls (similar in age and gestational age with singleton pregnancy) evaluated at time of delivery and at 6 months and 12 months postpartum. $p$ values below 0.05 are considered statistically significant. Results. The majority of the PE patients had persistence of proteinuria $(>120 \mathrm{mg} / \mathrm{L}$ after delivery) 6 months $(p=0.02)$ and 12 months postpartum ( $p<0.0001)$ compared to controls. Also reduced GFR (glomerular filtration rate) persisted up to 6 months postpartum in PE patients compared to controls $(p<0.001)$. Prepartum sFlt-1 levels indeed correlated with impaired renal function parameters. Conclusion. A significant proportion of our PE patients had lower GFR levels and persistent proteinuria up to 12 months postpartum. Prepartum sFlt-1 is a trend-setting marker for impaired renal function postpartum, but it is not sufficient enough to predict renal impairment after PE. An evaluation of 24 -month follow-up data is scheduled.

\section{Introduction}

Preeclampsia (PE) is a clinically severe and potentially life threatening complication of pregnancy. It is characterized by the onset of hypertension (systolic blood pressure $\geq 140 \mathrm{mmHg}$ or diastolic $\geq 90 \mathrm{mmHg}$ ) and proteinuria ( $\geq 300 \mathrm{mg} / 24$-hour collection) later than 20 weeks of gestation in a previously normotensive woman and occurs in about 2$8 \%$ of all pregnancies. It is a progressive disease and delivery cures the mother from symptoms, normally blood pressure normalizes, and proteinuria vanishes within few weeks until 6 weeks [1].
Its exact aetiology and pathophysiology still remain enigmatic [2]. Pathogenesis involves various molecular pathways resulting in endothelial dysfunction which impairs placental function and therefore fetal supply. In addition, maternal kidney endothelial damage leads to a decrease in renal blood flow, glomerular filtration rate (GFR) $[3,4]$, and proteinuria. The extent of proteinuria is considered as a marker of endothelial dysfunction and therefore of severity of preeclampsia $[1,4-6]$. However, microalbuminuria without impairment of renal function has been found several years after pregnancies complicated by PE $[7,8]$ suggesting persistent endothelial damage at least in some cases. 
In this context, epidemiologic studies have demonstrated that preeclampsia predisposes the mother to cardio- and cerebrovascular complications such as hypertension, stroke, or ischemic heart disease [9-13]. On the molecular side various factors also relevant for the development of hypertension in nonpregnant individuals show alterations in $\mathrm{PE}$ patients: antiangiogenic soluble fms-like tyrosine kinase-1 (sFlt-1, also known as sVEGFR1) is a naturally occurring, circulating antagonist to vascular endothelial growth factor (VEGF). Increased placental expression and secretion of sFlt-1 appear to play an important role in the pathogenesis of PE as sFlt1 can be found at increased levels in PE patients [14, 15]. The increase in sFlt-1 serum levels precedes clinical PE by about 5 weeks [16]. Proangiogenic placental growth factor (PlGF) belongs to the VEGF family and is also predominantly expressed in the placenta. PlGF increases in the first and second trimester and decreases in the third and is found at decreased levels in PE patients [17]. The ratio of these two biomarkers (sFlt-1/PlGF) has been recently established as an early predictive marker for the development of preeclampsia in the further course of the pregnancy [18]. Previous studies have discussed the use of the ratio as a possible predictive value to detect the development of preeclampsia, and a cut-off value of 85 had been determined [19]. For a cut-off value of 85 , the manufacturer gives a specificity of $95 \%$ and a sensitivity of $82 \%$ for their immunoassay.

The purpose of this prospective study was to investigate the predictive value of single prepartum findings indicating severity of preeclampsia for occurrence and extent of impaired postpartum kidney function. Furthermore we assessed the prognostic value of prepartum serum biomarkers sFlt-1 and PlGF for the development of persistent postpartum renal damage in PE patients.

\section{Patients and Methods}

In this prospective, single center study 68 patients with singleton pregnancy between 2012 and 2014 presenting at our department later than 20 weeks of gestational age with elevated blood pressure (systolic blood pressure $\geq$ $140 \mathrm{mmHg}$ or diastolic $\geq 90 \mathrm{mmHg}$ ) and confirmed proteinuria (>300 mg/24-hour collection) were screened.

44 women (Caucasian patients) with preeclampsia meeting inclusion criteria (diagnosis of PE, sufficient knowledge of German language, and having signed informed consent) were included in this study and compared to 39 healthy Caucasian controls (similar gestational week and patient's age). Exclusion criteria were preexisting renal or rheumatologic diseases or diabetes stated in the case history. Also all individuals with a history of preeclampsia in a previous pregnancy were excluded. Also all patients with relevant proteinuria documented in the maternity card before 20 weeks of gestation were excluded. In order to characterize maternal pre- and postpartum kidney function we measured various maternal (blood pressure, proteinuria, creatinine, carbamide, uric acid, serum protein, cystatin c, calcium, and GFR-CDKEPI) and fetal (birth weight) parameters. Data was collected in both groups not later than 24 hours before delivery. Mothers were scheduled for follow-up examinations at 6 and 12 months including blood pressure measurements and blood and urine analyses (24-hour collection and proteinuria threshold before delivery $<300 \mathrm{mg} / 24$-hour collection and after delivery $<120 \mathrm{mg} / \mathrm{L}$ ).

In PE patients maternal venous blood samples for sFlt-1 and PlGF measurements were collected immediately prior to delivery; serum was separated and stored at $-70^{\circ} \mathrm{C}$ until analysis. sFlt-1 and PlGF analysis were performed as an enzyme linked immunosorbent assay (ELISA) as recommended by the manufacturer (Roche Diagnostics, Mannheim, Germany).

This study was approved by the ethical board of HeinrichHeine-University Duesseldorf and informed consent was obtained from all subjects prior to enrolment.

2.1. Statistical Analysis. Data analysis was carried out using $\mathrm{R}$ (version 3.1.3) [20]. Boxplots have been created using $\mathrm{R}$ package ggplot2 [21]. $p$ values below 0.05 are considered statistically significant. The comparison between the PE patients and the controls was entered by the two-sample $t$-test. The correlations are done by Pearson's correlation coefficient. The evaluation of the $95 \%$ confidence interval of the correlations was done by Fisher's transformation.

Calculation of regression model for relationship between sFlt-1 and GFR (Figure 4(a)) was done in a two-step process. First, a nonparametric loess regression (using loess from package stats) was calculated allowing nonsupervised calculation of a nonlinear model. Confidence intervals were calculated from standard errors (obtained using predict (..., se $=$ TRUE)) and quantiles of $t$-distribution (as shown in Figure 4(a)). In a second step, a nonlinear least squares regression (NLS) (using nls from package stats) was calculated. An exponential relationship $(y=a \exp ((-x) / b))$ was assumed after visual fitting of model functions from which also starting parameter estimates $(a=8 \times 104, b=34)$ were derived. A numerical simplified version of the parameter estimates is provided. Predictions using simplified parameters differ from predictions using exact parameter estimates less than $1 \%$ for GFR $>25 \mathrm{~mL} / \mathrm{min} / 1.73 \mathrm{~m}^{2}$. The validity of the NLS derived model can be verified using the confidence intervals from loess regression.

\section{Results}

44 patients diagnosed with preeclampsia were compared with 39 gestational weeks and patient's age-matched controls. Compared to controls preeclampsia patients had a significantly increased proteinuria, higher serum creatinine, urea, uric acid, cystatin c, and elevated systolic and diastolic blood pressure. They also had significantly lower glomerular filtration rates (GFR-CDK-EPI), serum protein levels, and fetal birth weights (Table 1) at delivery.

3.1. A Proportion of PE Patients Display Persistent Proteinuria and Reduced Glomerular Filtration Rate 6 and 12 Months Postpartum. As indicators for impaired kidney function we investigated proteinuria and serum creatinine levels and glomerular filtration rate (GFR-CDK-EPI) (Figure 1). By definition all $\mathrm{PE}$ patients had increased proteinuria at delivery 


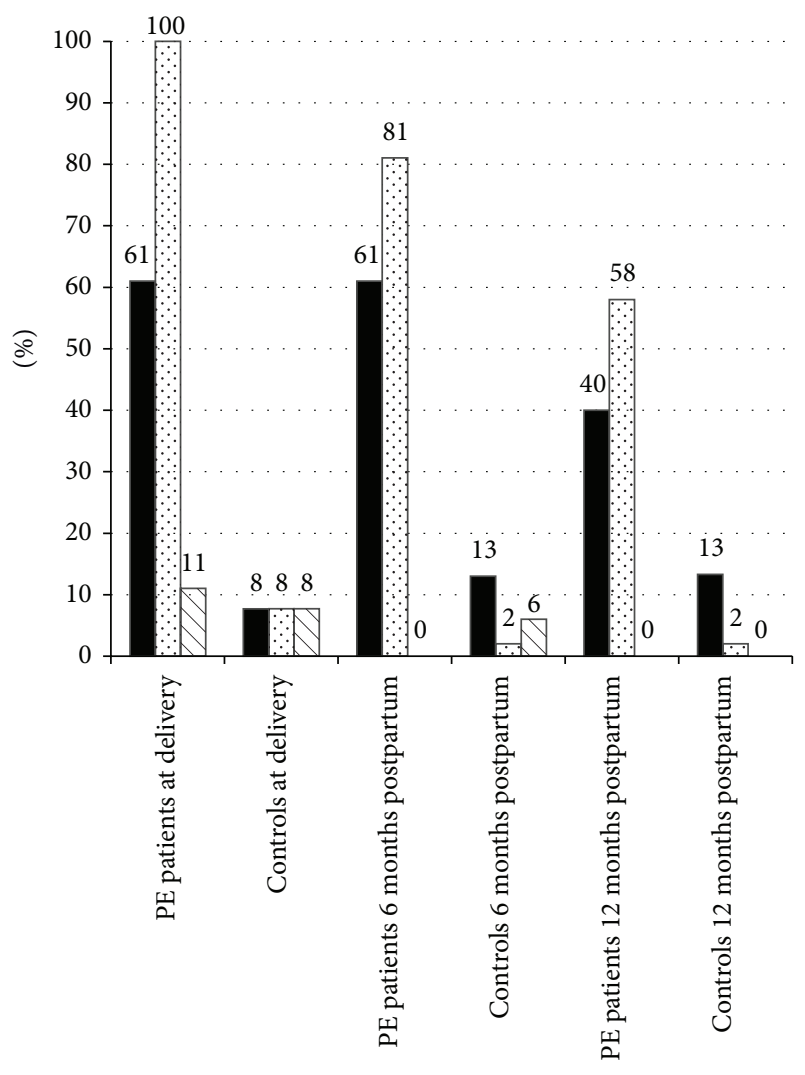

$\operatorname{GFR}\left(<90 \mathrm{~mL} / \mathrm{min} / 1.73 \mathrm{~m}^{2}\right)$

$\therefore$ Proteinuria ( $>300 \mathrm{mg} / 24$-hour collection before delivery or $>120 \mathrm{mg} / \mathrm{L}$ after delivery)

$\triangle$ Serum creatinine $(>79.2 \mu \mathrm{mol} / \mathrm{L})$

FIGURE 1: Distribution of impaired renal function parameters (GFR, proteinuria, and serum creatinine) in PE patients and controls at delivery, 6 months postpartum, and 12 months postpartum.

(>300 mg/24-hour collection), whereas it was diagnosed in only $3 / 39(7.7 \%)$ of controls $(3 / 39)(p<0.0001)$. Reduced GFR $<90 \mathrm{~mL} / \mathrm{min} / 1.73 \mathrm{~m}^{2}$ at delivery was found in $27 / 44$ $(61 \%)$ of PE patients and in $3 / 39(7.7 \%)$ of controls $(p<$ $0.0001)$. Serum creatinine was increased $>79.2 \mu \mathrm{mol} / \mathrm{L}$ in $5 / 44(11 \%)$ of patients and in $3 / 39(7.7 \%)$ of controls $(p=$ $0.72)$.

A persisting proteinuria $(>120 \mathrm{mg} / \mathrm{L})$ was found in $29 / 36$ (81\%) of PE patients after 6 months (versus $1 / 39$ (2\%) of controls, $p=0.02$ ) and 14/24 (58\%) after 12 months (versus $2 / 30(1 \%)$ of controls, $p<0.0001)$. The GFR reduction was found in 22/36 (61\%) of PE patients after 6 (versus 5/39 (13\%) of controls, $p<0.0001)$ and in $10 / 25(40 \%)$ of PE patients after 12 months (versus $4 / 30(13.3 \%)$ of controls, $p=0.21$ ), respectively.

\subsection{Proteinuria and GFR Show a Positive Pre- and Postpartum} Correlation. Next we identified predictors of persistently impaired kidney function after 6 and 12 months. Prepartum proteinuria was positively correlated with proteinuria at 6 $(r=0.74 ; 95 \% \mathrm{CI}+0.54$ to +0.87$)$ and 12 months postpartum $(r=0.55 ; 95 \% \mathrm{CI}+0.09$ to +0.81$)$.
We found also a positive correlation between pre- and postpartum GFR in patients after $6(r=0.73 ; 95 \% \mathrm{CI}+0.51$ to +0.86$)$ and 12 months of $(r=0.49 ; 95 \% \mathrm{CI}+0.01$ to +0.78 ) follow-up (Figure 2). However, prepartum GFR and proteinuria were not correlated $(r=-0.11 ; 95 \%$ CI -0.39 to +0.20$)$. Also, prepartum GFR was not correlated with postpartum proteinuria after $6(r=-0.22$; $95 \% \mathrm{CI}-0.60$ to +0.34$)$ or 12 months $(r=-0.17 ; 95 \% \mathrm{CI}-0.61$ to +0.34$)$, respectively.

The amount of proteinuria at delivery ranged between 300 and 12133 mg/24-hour collection. Proteinuria exceeding $2000 \mathrm{mg} / 24$-hour collection was restricted to PE patients with a GFR $<104 \mathrm{~mL} / \mathrm{min} / 1.73 \mathrm{~m}^{2}$ and was not found in control individuals.

3.3. Negative Relationship between sFlt-1 and GFR. Prepartum clinical parameters were analysed in order to prove their ability to predict impaired postpartum kidney function. Mean arterial pressure $(\mathrm{MAP}=$ diastolic blood pressure $+1 / 3 *$ (systolic blood pressure - diastolic blood pressure)) decreased from $109 \mathrm{mmHg}( \pm 9.6 \mathrm{SD})$ prepartum to $100 \mathrm{mmHg}( \pm 10.6 \mathrm{SD})$ at 6 months postpartum $(r=0.31 ; 95 \%$ $\mathrm{CI}-0.04$ to +0.59$)$ and to $91 \mathrm{mmHg}( \pm 13.6 \mathrm{SD})$ at 12 months postpartum ( $r=0.25$; $95 \% \mathrm{CI}-0.26$ to +0.65$)$ in PE patients. In the controls MAP remained unchanged (Figure 3 ).

Then we investigated if sFlt-1 or PlGF alone or in combination with each other or other clinical parameters are good predictors of persistent postpartum renal impairment in $\mathrm{PE}$ patients. The comparison between GFR and sFlt-1 revealed a significant inverse relationship ( $r=-0.63 ; 95 \% \mathrm{CI}-0.78$ to -0.40 ). Figure 4(a) shows a relationship between sFlt-1 and GFR values. From nonlinear least squares regression using an exponential model $(y=a \exp (-x / b))$ we derived the model

$$
y=68170 e^{(-x / 39)}
$$

for relationship between $y=$ sFlt-1 $(\mathrm{pg} / \mathrm{mL})$ and $x=$ GFR $\left(\mathrm{mL} / \mathrm{min} / 1.73 \mathrm{~m}^{2}\right)$ using simplified numeric values. Predicted values for sFlt-1 from this model are shown in Figure 4(a) (solid line).

The analysis between PlGF and GFR demonstrated no statistic relevant relationship (Figure 4(b), $r=0.16$; 95\% CI -0.14 to +0.44$)$ and also the analysis between the ratio sFlt1/PlGF and GFR (Figure 4(c)).

The biomarkers sFlt-1 and PlGF and proteinuria immediately prior to delivery were only weakly correlated $(r=-0.05$; $95 \%$ CI -0.34 to +0.25 ).

In our sample an inverse relationship between sFlt-1 and GFR is present; therefore we analysed all patients with reduced GFR, defined as a GFR below $90 \mathrm{~mL} / \mathrm{min} / 1.73 \mathrm{~m}^{2}$. sFlt- 1 alone reveals a sensitivity of $90 \%$ and a specificity of $31 \%$ at a value below $5000 \mathrm{pg} / \mathrm{mL}$ as a predictive marker for reduced GFR after 6 months postpartum. sFlt-1 values $>5000 \mathrm{pg} / \mathrm{mL}$ reach meaningful specificities with loss of sensitivity as predictive marker for impaired renal function (Figures 5(a) and 5(b)). Figures 5(c) and 5(d) show also the specificities and sensitivities of sFlt-1 combined with MAP > $110 \mathrm{mmHg}$ as predictive markers for impaired renal 
TABLE 1: Baseline characteristics of preeclampsia patients and controls immediately before delivery (maximum 24 hours before delivery). Comparison entered by two-sample $t$-test.

\begin{tabular}{|c|c|c|c|c|}
\hline & PE patients $(n=44)^{*}$ & Controls $(n=39)^{*}$ & Reference values & $p$ value \\
\hline Delivery age (y) & $31.95[22-43]$ & $31.40[23-42]$ & & 0.64 \\
\hline Gestational age (d) & 243 [189-278] & 245 [195-271] & & 0.65 \\
\hline Birth weight (g) & $2336[1160-3480]$ & 2720 [1570-3919] & & 0.003 \\
\hline Proteinuria & $2639.9[301-12133]$ & $180.9[60-891.5]$ & $\begin{array}{c}<300 \mathrm{mg} / 24 \mathrm{~h} \text { in } \\
\text { pregnancy } \\
<120 \mathrm{mg} / \mathrm{L} \text { outside } \\
\text { of pregnancy }\end{array}$ & $<0.0001$ \\
\hline Creatinine $(\mu \mathrm{mol} / \mathrm{L})$ & $66.88[35.2-96.8]$ & $52.8[17.6-105.6]$ & $<0.9$ & 0.001 \\
\hline Urea (mg/dL) & $27.41[10-54]$ & $33.21[14-55]$ & $21-43$ & 0.018 \\
\hline Uric acid $(\mathrm{mg} / \mathrm{dL})$ & $6.01[4.3-8.8]$ & $5.51[4.1-7.5]$ & $<5.7$ & 0.023 \\
\hline GFR-CDK-EPI $\left(\mathrm{mL} / \mathrm{min} / 1.73 \mathrm{~m}^{2}\right)$ & $79.32[29-127]$ & $113.05[71-198]$ & $90-140$ & $<0.0001$ \\
\hline Serum protein $(\mathrm{g} / \mathrm{dL})$ & $5.27[3.87-6.72]$ & $6.35[4.7-8.3]$ & $6.40-8.30$ & $<0.0001$ \\
\hline Cystatin C (mg/L) & $1.31[0.8-2.33]$ & $0.74[0.66-0.89]$ & $0.6-1.1$ & $<0.0001$ \\
\hline Calcium (mmol/L) & $2.12[1.7-3.8]$ & $2.08[1.85-2.51]$ & $2.20-2.55$ & 0.508 \\
\hline sFlt-1 (pg/mL) & $10708[635-33333]$ & - & - & - \\
\hline PlGF (pg/mL) & $67.9[5.02-421.5]$ & - & - & - \\
\hline Systolic blood pressure (mmHg) & $150.95[128-170]$ & $125.26[89-145]$ & $<140$ & $<0.0001$ \\
\hline Diastolic blood pressure (mmHg) & $88.79[67-110]$ & $76.64[45-92]$ & $<90$ & $<0.0001$ \\
\hline
\end{tabular}

${ }^{*}$ All values are presented as mean [range].
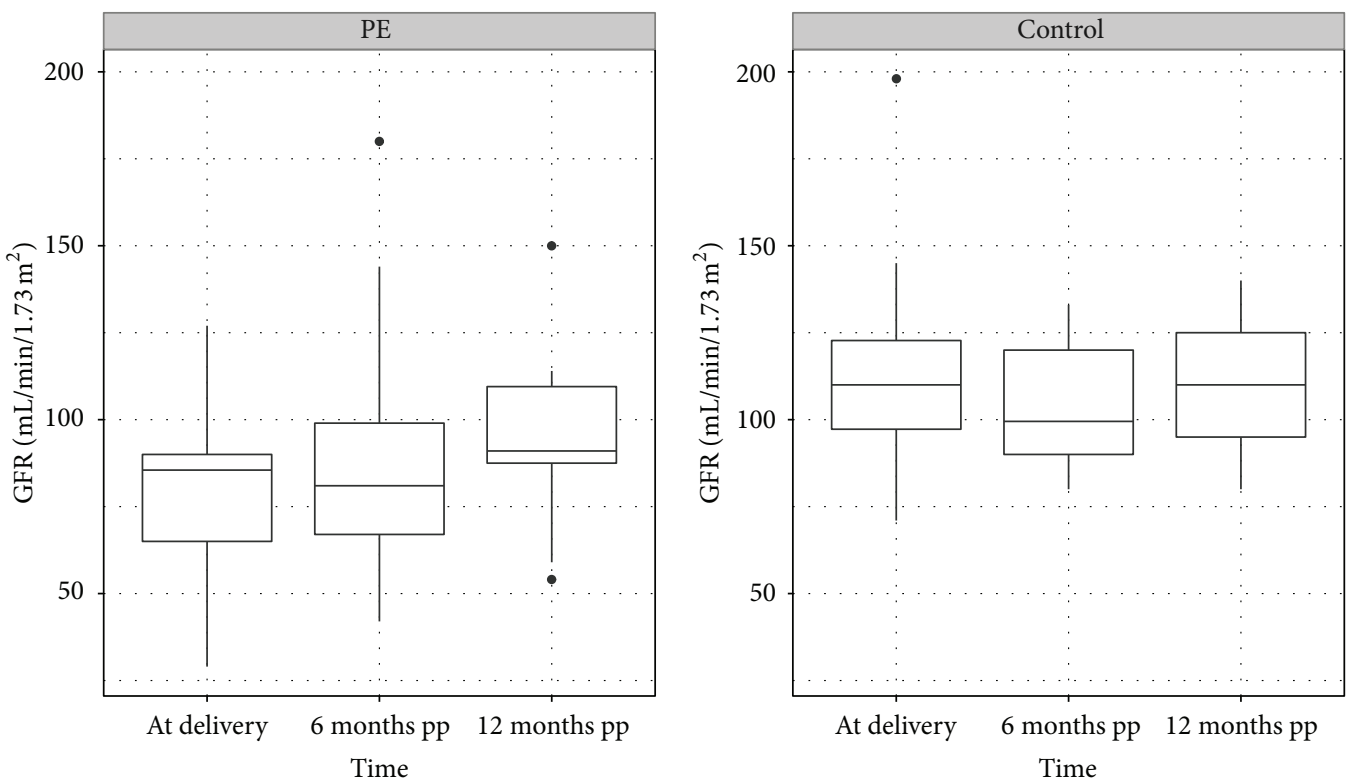

FIGURE 2: Distribution of GFR in PE patients and controls at delivery, 6 months postpartum, and 12 months postpartum (pp). PE patients: mean GFR $79.3 \mathrm{~mL} / \mathrm{min} / 1.73 \mathrm{~m}^{2}( \pm 23.2 \mathrm{SD})$ immediately at delivery, $85.4 \mathrm{~mL} / \mathrm{min} / 1.73 \mathrm{~m}^{2}$ ( $\left.\pm 28.4 \mathrm{SD}\right) 6$ months postpartum, and $95.3 \mathrm{~mL} / \mathrm{min} / 1.73 \mathrm{~m}^{2}( \pm 23.2 \mathrm{SD}) 12$ months postpartum. Controls: mean GFR $113.0 \mathrm{~mL} / \mathrm{min} / 1.73 \mathrm{~m}^{2}( \pm 22.7 \mathrm{SD})$ at delivery, $104.0 \mathrm{~mL} / \mathrm{min} / 1.73 \mathrm{~m}^{2}( \pm 15.0 \mathrm{SD}) 6$ months postpartum, and $110.7 \mathrm{~mL} / \mathrm{min}( \pm 19.3 \mathrm{SD}) 12 \mathrm{months}$ postpartum (pp). 

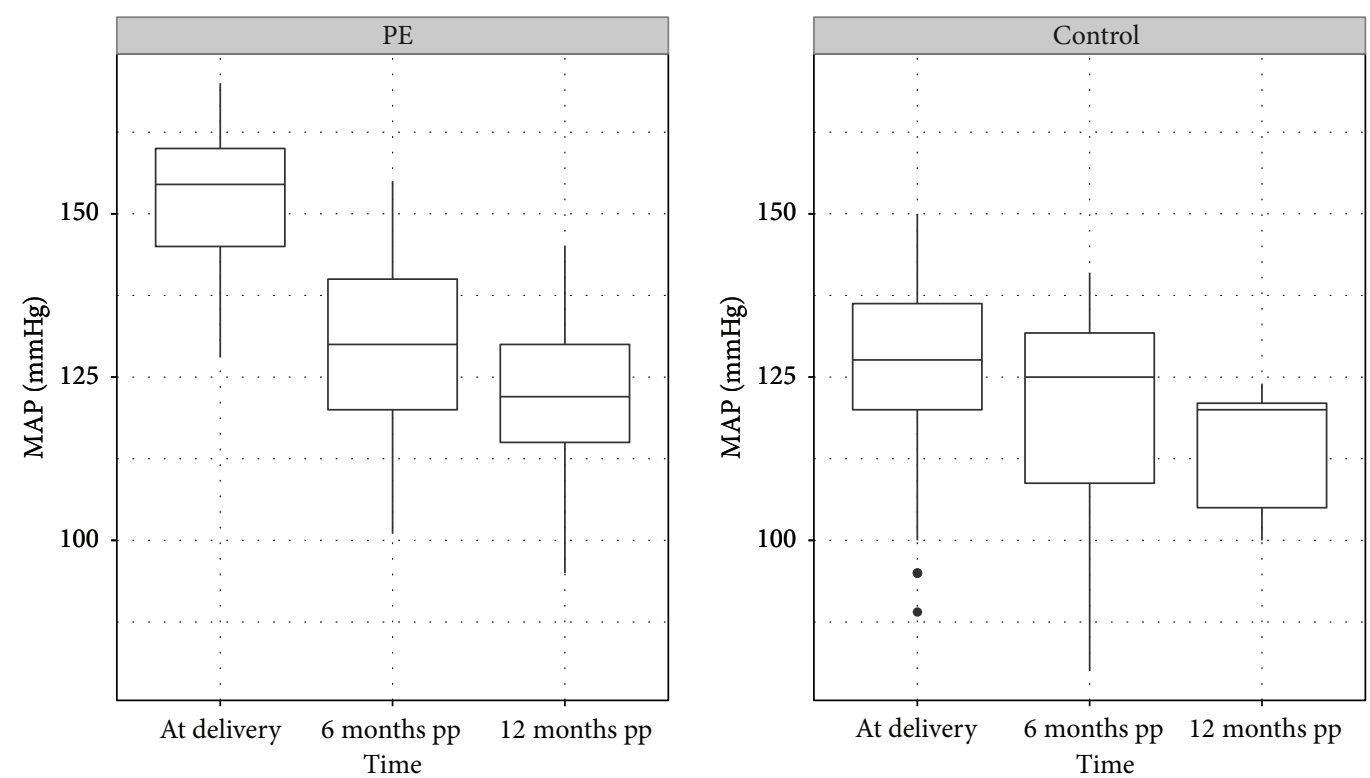

FIGURE 3: Distribution of mean arterial pressure values (MAP) in controls and PE patients. PE patients at delivery $109 \mathrm{mmHg}( \pm 9.6 \mathrm{SD})$, 6 months postpartum (pp) $100 \mathrm{mmHg}( \pm 10.6 \mathrm{SD})$, and 12 months postpartum $(\mathrm{pp}) 91 \mathrm{mmHg}( \pm 13.6 \mathrm{SD})$. Controls: at delivery $92 \mathrm{mmHg}$ ( $\pm 12.6 \mathrm{SD}), 6$ months postpartum (pp) $93 \mathrm{mmHg}( \pm 13.0 \mathrm{SD})$, and 12 postpartum (pp) $95 \mathrm{mmHg}( \pm 7.6 \mathrm{SD})$.

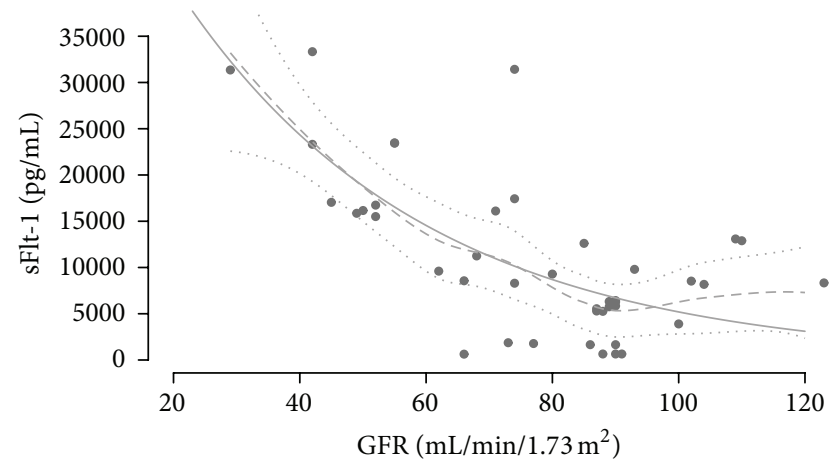

(a)

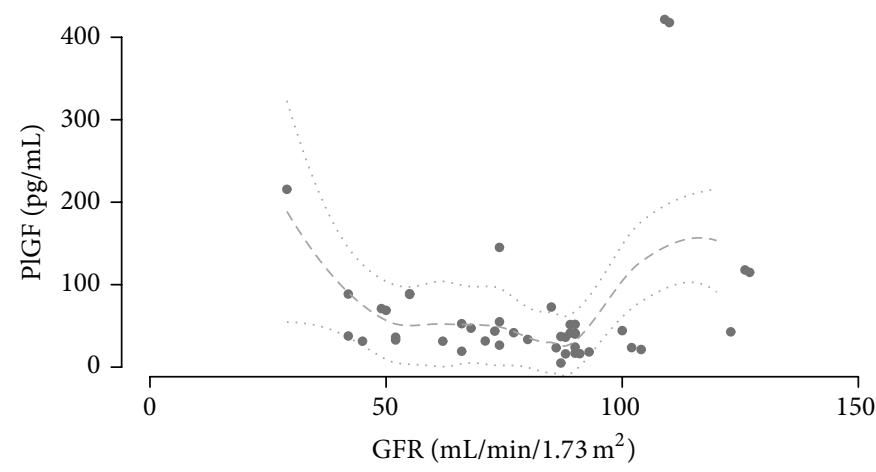

(b)

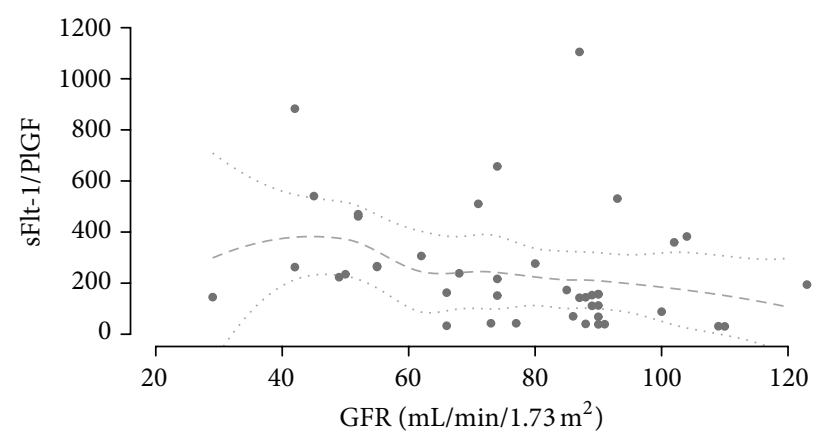

(c)

FIGURE 4: (a) Distribution of sFlt-1 in relation to GFR in PE patients. Predicted values from loess regression are shown together with 95\% confidence intervals (discontinuous lines). Predicted values from NLS regression $(y=a \exp (-x / b))$ are shown in solid line. Estimated parameter values were $a=68169.27 b=38.84402$. (b) Distribution of PlGF in relation to GFR in PE patients. Predicted values from loess regression are shown together with $95 \%$ confidence intervals (discontinuous lines). (c) Distribution of sFlt-1/PlGF-ratio in relation to GFR in PE patients. Predicted values from loess regression are shown together with 95\% confidence intervals (discontinuous lines). 


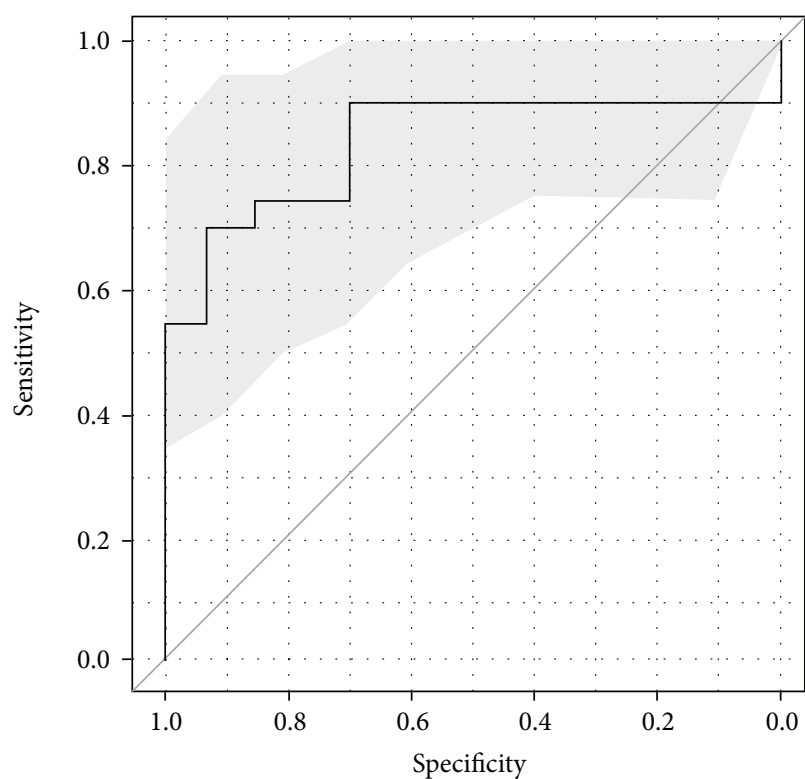

(a)

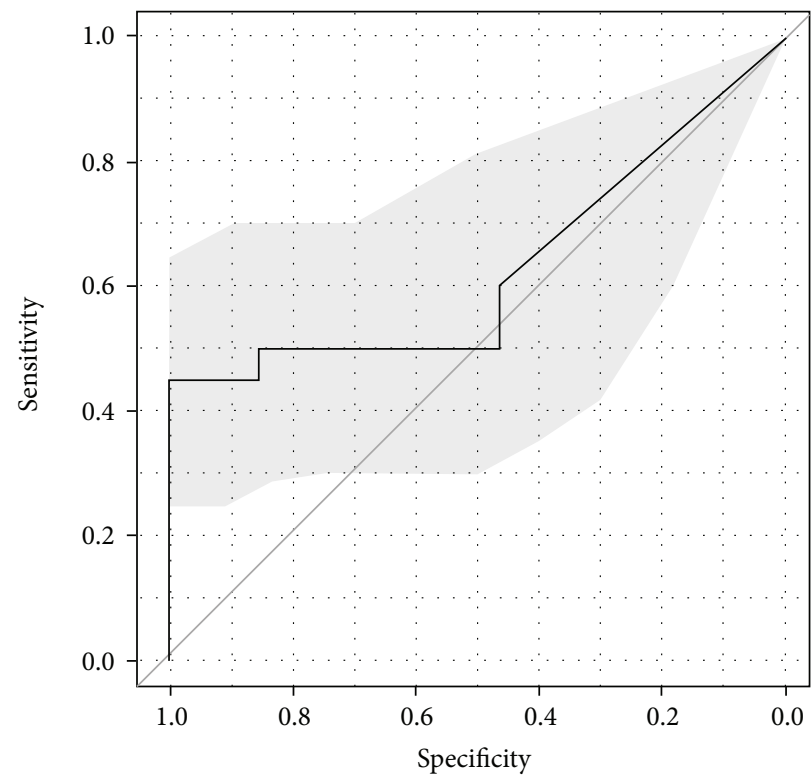

(c)

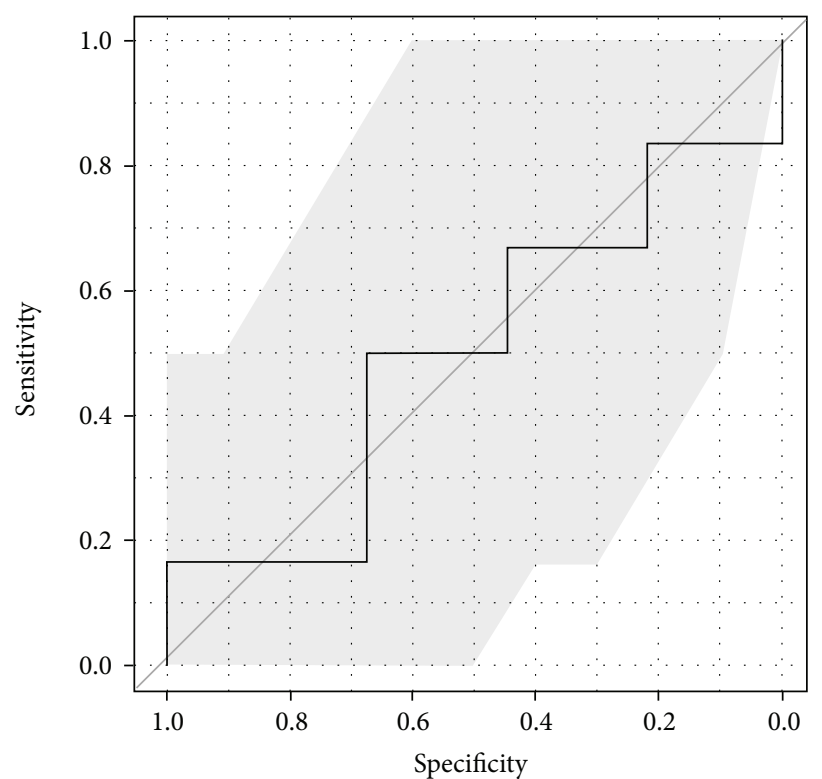

(b)

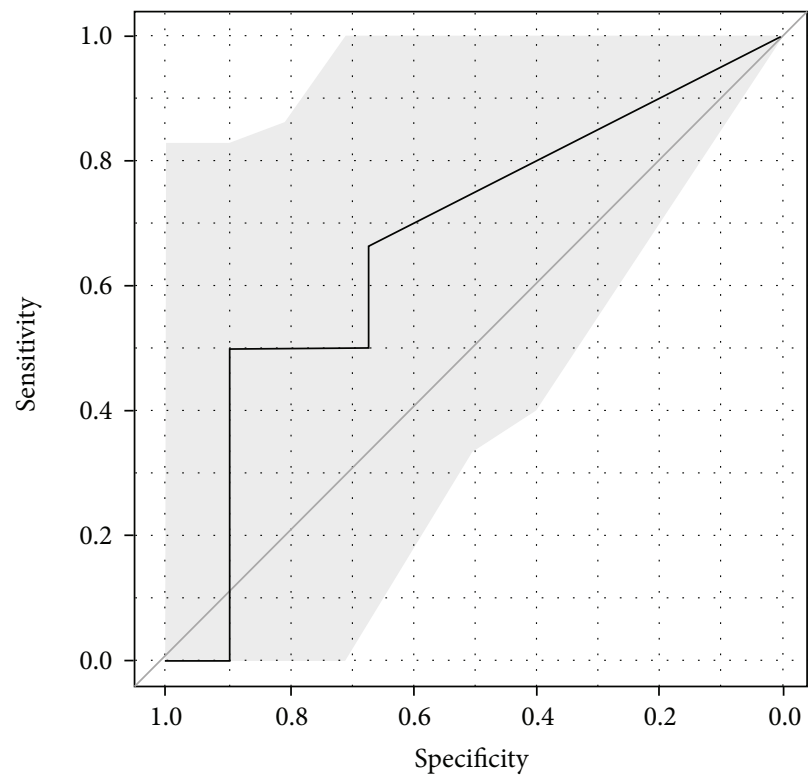

(d)

FIGURE 5: (a) ROC curve for sFlt-1 as a predictive marker for impaired renal function (defined as GFR $<90 \mathrm{~mL} / \mathrm{min} / 1.73 \mathrm{~m}{ }^{2}$ ) $6 \mathrm{months}$ after delivery in PE patients. The grey shaded area shows the $95 \%$ confidence interval. AUC $=0.83$. (b) ROC curve for sFlt- 1 as a predictive marker for impaired renal function (defined as GFR $<90 \mathrm{~mL} / \mathrm{min} / 1.73 \mathrm{~m}^{2}$ ) 12 months after delivery in PE patients. The grey shaded area shows the $95 \%$ confidence interval. AUC $=0.5$. (c) ROC curve for sFlt-1 and MAP $>110 \mathrm{mmHg}$ as a predictive marker for impaired renal function (defined as GFR $\left.<90 \mathrm{~mL} / \mathrm{min} / 1.73 \mathrm{~m}^{2}\right) 6$ months after delivery in PE patients. The grey shaded area shows the $95 \%$ confidence interval. AUC $=0.63$. (d) ROC curve for sFlt-1 and MAP $>110 \mathrm{mmHg}$ as a predictive marker for impaired renal function (defined as GFR $<90 \mathrm{~mL} / \mathrm{min} / 1.73 \mathrm{~m}{ }^{2}$ ) 12 months after delivery in PE patients. The grey shaded area shows the 95\% confidence interval. AUC $=0.67$.

function (GFR $\left.<90 \mathrm{~mL} / \mathrm{min} / 1.73 \mathrm{~m}^{2}\right)$. PlGF did not show any correlation with the investigated parameters.

\section{Discussion}

Improvements in the management of PE patients have lowered fetal and maternal morbidity and mortality caused by PE. Second trimester sFlt-1/PlGF-ratio has the potential to predict $\mathrm{PE}$ in asymptomatic patients and therefore to allow early treatment. Early medical treatment and fetal monitoring help to prolong pregnancies in order to avoid increased fetal mortality and morbidity related to preterm delivery and therefore fetal immaturity. In some cases this takes into account that under prolongation of pregnancy PE 
progresses and therefore maternal morbidity increases. As PE is progressive and delivery cures symptoms for the mother it is crucial to investigate if severity of PE persistently affects mothers' health.

Physiologically, during pregnancy the renal plasma flux and GFR increase until the end of first trimester by $50 \%$ and normalize 3 months following delivery [22]. In our controls we also find a moderate increase in GFR during normal pregnancy. In PE placental factors lead to microangiopathy, arterial constriction, microangiopathy, and finally reduced GFR. As expected a significant proportion (61\%) of our PE patients had lower GFR levels at delivery, which in $40 \%$ persisted up to 12 months after delivery.

One consequence of endothelial dysfunction in $\mathrm{PE}$ is the progressive proteinuria, which itself is also an indicator for impaired kidney function beyond pregnancy. In a metaanalysis McDonald et al. combined data from seven cohorts and confirmed the association between $\mathrm{PE}$ and persisting albuminuria [23]. At an average of 7.1 years of follow-up, $31 \%$ of women who had preeclampsia developed microalbuminuria in contrast to only $7 \%$ with uncomplicated pregnancies [23]. In our study also persistent proteinuria was found in $58 \%$ of $\mathrm{PE}$ patients at least 12 months after delivery but only in $1 \%$ of controls. Two other studies showed an increased risk of renal disease after PE [24, 25]. 7.7\% of the controls had a relevant proteinuria prepartum but without increase of blood pressure. This proportion is conforming to the present data $[24,25]$. There is also evidence that $\mathrm{PE}$ is a risk factor for later development of hypertension and cardiovascular diseases [26, 27]. In contrast, persisting proteinuria is not necessarily associated with increased blood pressure or persisting microalbuminuria [28]. Interestingly, in our study sFlt-1 levels correlate with impaired renal function parameters but do not correlate with the extent of proteinuria before delivery of a PE patient compared with healthy controls similar in gestational age or patient's age.

Critically high blood pressure in PE despite medical therapy can be a reason for preterm delivery. This might explain the correlation between increased prepartum blood pressure and lower birth weight and gestational age. High blood pressure during PE pregnancy can also persist [29].

sFlt-1 levels are directly correlated with severity of PE and inversely correlated with time to onset of proteinuria and hypertension in preeclampsia patients [30]. High sFlt-1 levels persist after delivery in preeclampsia patients [31]. We aimed to investigate if sFlt-1 can serve as a predictive marker for persistent endothelial dysfunction and impairment of renal function. There are no sFlt-1 or PlGF measurements in controls prior to delivery, because both parameters are evaluated in preeclampsia patients [19]. We demonstrated a relationship between increased sFlt-1 levels and decreased GFR in PE patients. sFlt-1 is a sensitive prognostic marker for reduced GFR 6 months postpartum below a value of $10000 \mathrm{pg} / \mathrm{mL}$. sFlt-1 values above $10000 \mathrm{pg} / \mathrm{mL}$ are attended by a maximum specificity, but lower sensitivity (6 months postpartum). sFlt1 level until $5000 \mathrm{pg} / \mathrm{mL}$ and MAP > $110 \mathrm{mmHg}$ have the most meaningful sensitivities and specificities as predictive markers for impaired renal function (GFR $\left.<90 \mathrm{~mL} / \mathrm{min} / 1.73 \mathrm{~m}^{2}\right) 6$ and 12 months postpartum. Overall in our patients collective sFlt-1 is a trend-setting marker in PE, but it is not sufficient enough to predict persistent endothelial dysfunction and impairment of renal function.

Neither sFlt-1 and blood pressure nor prepartum proteinuria alone can predict the extent of renal impairment after delivery. sFlt-1 is indeed a trend-setting marker in PE. Particularly sFlt-1 values until $10000 \mathrm{pg} / \mathrm{mL}$ are sensitive to predicting a renal impairment after preeclampsia. Higher values are specific but without sufficient sensitivity. Further investigations are needed to evaluate the prediction of a persisting renal impairment after preeclampsia with angiogenic growth factor sFlt-1.

Limitations of our study are the relatively low number of patients. In an analysis of $30 \mathrm{PE}$ patients with low $(<5 \mathrm{~g} /$ day $)$ or high ( $>5 \mathrm{~g} /$ day) amounts of proteinuria, proteinuria itself was not correlated with kidney function parameters 5-6 years after pregnancy complicated by PE [32]. We demonstrate a persistence of a clinical relevant proteinuria up to 1 year after delivery in patients with PE. In this respect our study is limited as long term follow-up data is not available. However, an evaluation of 24-month follow-up data is scheduled.

\section{Ethical Approval}

This study was approved by the ethical board of HeinrichHeine-University Duesseldorf.

\section{Competing Interests}

None of the authors have declared financial competing interests.

\section{Authors' Contributions}

T. Kaleta interpreted the analysis, drafted the paper, and substantially revised it. A. Stock performed the experiments. D. Panayotopoulos participated substantially in paper revision. O. Vonend substantially revised the paper. D. Niederacher contributed reagents, interpreted analysis, and substantially revised the paper. M. Neumann analysed the data. T. Fehm substantially revised the paper. W. Kaisers participated substantially in data interpretation and paper revision. M. Fleisch designed the study, interpreted the analysis, drafted the paper, and substantially revised the paper.

\section{Acknowledgments}

The observational study described in this paper was funded by own resources of the Department of Gynaecology and Obstetrics of the University Hospital of Duesseldorf.

\section{References}

[1] S. Chua and C. W. G. Redman, "Prognosis for pre-eclampsia complicated by $5 \mathrm{~g}$ or more of proteinuria in 24 hours," European Journal of Obstetrics and Gynecology and Reproductive Biology, vol. 43, no. 1, pp. 9-12, 1992. 
[2] C. W. Redman and I. L. Sargent, "Latest advances in understanding preeclampsia," Science, vol. 308, no. 5728, pp. 1592-1594, 2005.

[3] S. A. Karumanchi, S. E. Maynard, I. E. Stillman, F. H. Epstein, and V. P. Sukhatme, "Preeclampsia: a renal perspective," Kidney International, vol. 67, no. 6, pp. 2101-2113, 2005.

[4] P. Moran, M. D. Lindheimer, and J. M. Davison, "The renal response to preeclampsia," Seminars in Nephrology, vol. 24, no. 6, pp. 588-595, 2004.

[5] P. Chan, M. Brown, J. M. Simpson, and G. Davis, "Proteinuria in pre-eclampsia: how much matters?" BJOG: An International Journal of Obstetrics and Gynaecology, vol. 112, no. 3, pp. 280285, 2005.

[6] R. Lafayette, "The kidney in preeclampsia," Kidney International, vol. 67, no. 3, pp. 1194-1203, 2005.

[7] J. Bar, B. Kaplan, C. Wittenberg et al., "Microalbuminuria after pregnancy complicated by pre-eclampsia," Nephrology Dialysis Transplantation, vol. 14, no. 5, pp. 1129-1132, 1999.

[8] H. Nisell, H. Lintu, N. O. Lunell, G. Mollerstrom, and E. Pettersson, "Blood pressure and renal function seven years after pregnancy complicated by hypertension," British Journal of Obstetrics and Gynaecology, vol. 102, no. 11, pp. 876-881, 1995.

[9] C. V. Ananth, M. R. Peltier, W. L. Kinzler, J. C. Smulian, and A. M. Vintzileos, "Chronic hypertension and risk of placental abruption: is the association modified by ischemic placental disease?" American Journal of Obstetrics \& Gynecology, vol. 197, no. 3, pp. 273.el-273.e7, 2007.

[10] C. V. Ananth, M. R. Peltier, M. R. Chavez, R. S. Kirby, D. Getahun, and A. M. Vintzileos, "Recurrence of ischemic placental disease," Obstetrics and Gynecology, vol. 110, no. 1, pp. 128-133, 2007.

[11] S. Baumwell and S. A. Karumanchi, "Pre-eclampsia: clinical manifestations and molecular mechanisms," Nephron-Clinical Practice, vol. 106, no. 2, pp. c72-c81, 2007.

[12] J. T. Drost, A. H. E. M. Maas, J. van Eyck, and Y. T. van der Schouw, "Preeclampsia as a female-specific risk factor for chronic hypertension," Maturitas, vol. 67, no. 4, pp. 321-326, 2010.

[13] H. U. Irgens, L. Reisæter, L. M. Irgens, and R. T. Lie, "Long term mortality of mothers and fathers after pre-eclampsia: population based cohort study," British Medical Journal, vol. 323, no. 7323, pp. 1213-1216, 2001.

[14] A. Rajakumar, H. M. Michael, P. A. Rajakumar et al., "Extraplacental expression of vascular endothelial growth factor receptor-1, (Flt-1) and soluble Flt-1 (sFlt-1), by peripheral blood mononuclear cells (PBMCs) in normotensive and preeclamptic pregnant women," Placenta, vol. 26, no. 7, pp. 563-573, 2005.

[15] P. Vuorela, S. Helske, C. Hornig, K. Alitalo, H. Weich, and E. Halmesmäki, "Amniotic fluid-soluble vascular endothelial growth factor receptor-1 in preeclampsia," Obstetrics and Gynecology, vol. 95, no. 3, pp. 353-357, 2000.

[16] R. J. Levine, S. E. Maynard, C. Qian et al., "Circulating angiogenic factors and the risk of preeclampsia," The New England Journal of Medicine, vol. 350, no. 7, pp. 672-683, 2004.

[17] C. J. Robinson and D. D. Johnson, "Soluble endoglin as a second-trimester marker for preeclampsia," American Journal of Obstetrics and Gynecology, vol. 197, no. 2, pp. 174-175, 2007.

[18] R. J. Levine, C. Lam, C. Qian et al., "Soluble endoglin and other circulating antiangiogenic factors in preeclampsia," The New England Journal of Medicine, vol. 355, no. 10, pp. 992-1005, 2006.
[19] H. Stepan, W. Schaarschmidt, A. Jank, S. Verlohren, and J. Kratzsch, "Use of angiogenic factors (sFlt-1/PlGF ratio) to confirm the diagnosis of preeclampsia in clinical routine: first experience," Zeitschrift fur Geburtshilfe und Neonatologie, vol. 214, no. 6, pp. 234-238, 2010.

[20] R Core Team, R: A Language and Environment for Statistical Computing, R Foundation for Statistical Computing, Vienna, Austria, 2015, http://www.R-project.org/.

[21] H. Wickham, Ggplot2: Elegant Graphics for Data Analysis, Springer, New York, NY, USA, 2009.

[22] J. Airoldi and L. Weinstein, "Clinical significance of proteinuria in pregnancy," Obstetrical and Gynecological Survey, vol. 62, no. 2, pp. 117-124, 2007.

[23] S. D. McDonald, Z. Han, M. W. Walsh, H. C. Gerstein, and P. J. Devereaux, "Kidney disease after preeclampsia: a systematic review and meta-analysis," American Journal of Kidney Diseases, vol. 55, no. 6, pp. 1026-1039, 2010.

[24] I.-K. Wang, C.-H. Muo, Y.-C. Chang et al., "Association between hypertensive disorders during pregnancy and end-stage renal disease: a population-based study," Canadian Medical Association Journal, vol. 185, no. 3, pp. 207-213, 2013.

[25] B. E. Vikse, L. M. Irgens, T. Leivestad, R. Skjærven, and B. M. Iversen, "Preeclampsia and the risk of end-stage renal disease," The New England Journal of Medicine, vol. 359, no. 8, pp. 800809, 2008.

[26] E. B. Magnussen, L. J. Vatten, G. D. Smith, and P. R. Romundstad, "Hypertensive disorders in pregnancy and subsequently measured cardiovascular risk factors," Obstetrics and Gynecology, vol. 114, no. 5, pp. 961-970, 2009.

[27] J. W. Rich-Edwards, T. F. McElrath, S. A. Karumanchi, and E. W. Seely, "Breathing life into the lifecourse approach: pregnancy history and cardiovascular disease in women," Hypertension, vol. 56, no. 3, pp. 331-334, 2010.

[28] M. K. Sandvik, S. Hallan, E. Svarstad, and B. E. Vikse, "Preeclampsia and prevalence of microalbuminuria 10 years later," Clinical Journal of the American Society of Nephrology, vol. 8, no. 7, pp. 1126-1134, 2013.

[29] R. A. Costa Sr., V. Dos Santos Silva, D. B. Dias, R. P. da Rocha, L. C. Martin, and J. C. Peraçoli, "Postpartum evaluation of blood pressure and proteinuria in women with severe preeclampsia," Pregnancy Hypertens, vol. 5, no. 1, p. 71, 2015.

[30] S. A. Karumanchi and M. D. Lindheimer, "Preeclampsia pathogenesis: 'Triple a rating'-Autoantibodies and antiAngiogenic factors," Hypertension, vol. 51, no. 4, pp. 991-992, 2008.

[31] M. Wolf, C. A. Hubel, C. Lam et al., "Preeclampsia and future cardiovascular disease: potential role of altered angiogenesis and insulin resistance," Journal of Clinical Endocrinology and Metabolism, vol. 89, no. 12, pp. 6239-6243, 2004.

[32] K. H. Lampinen, M. Rönnback, P.-H. Groop, and R. J. Kaaja, "Renal and vascular function in women with previous preeclampsia: a comparison of low- and high-degree proteinuria," Kidney International, vol. 70, no. 10, pp. 1818-1822, 2006. 


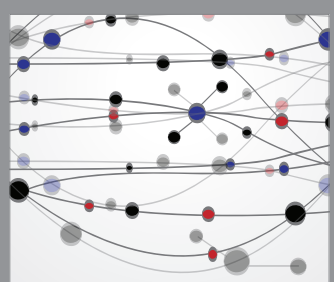

The Scientific World Journal
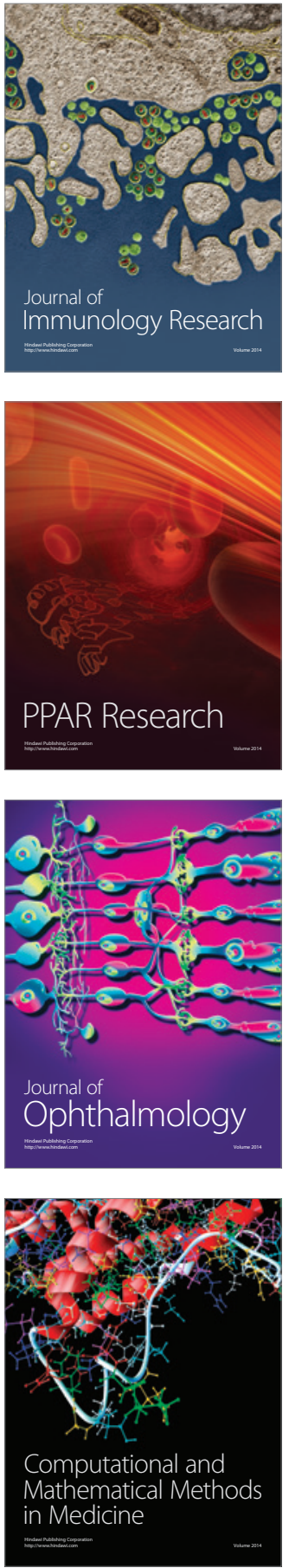

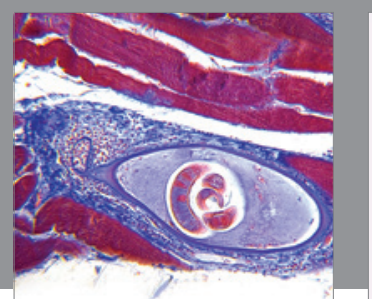

Gastroenterology Research and Practice

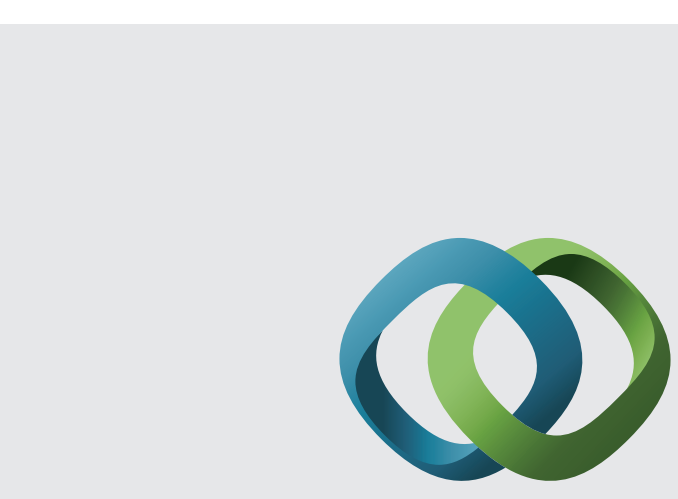

\section{Hindawi}

Submit your manuscripts at

http://www.hindawi.com
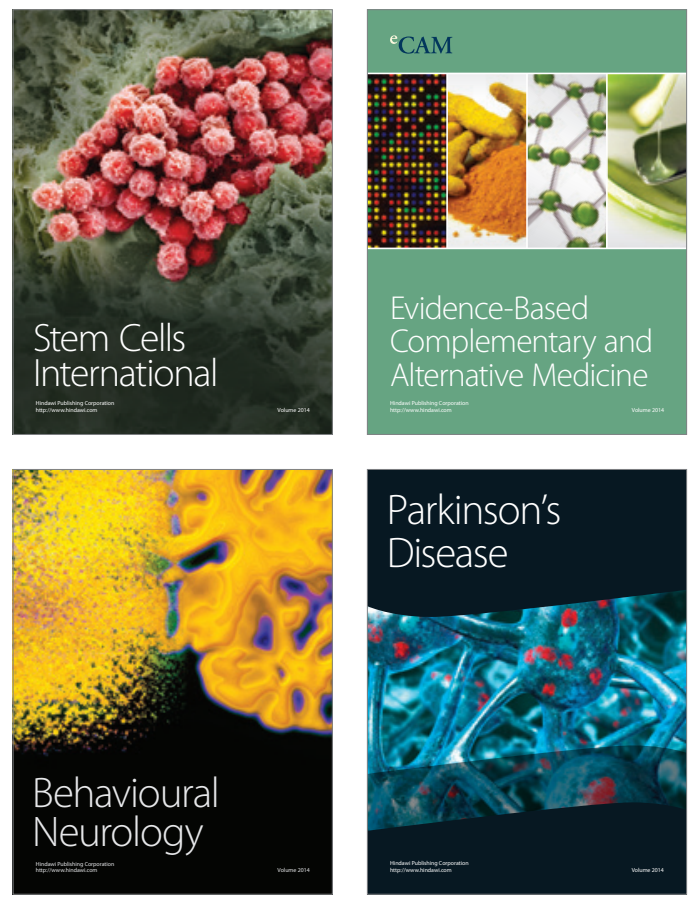
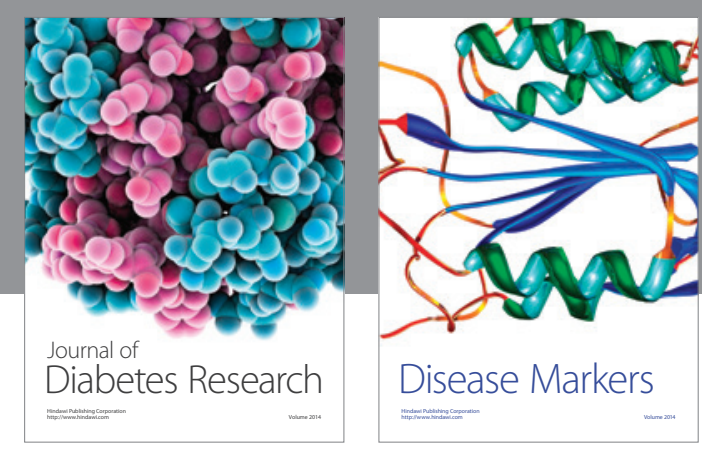

Disease Markers
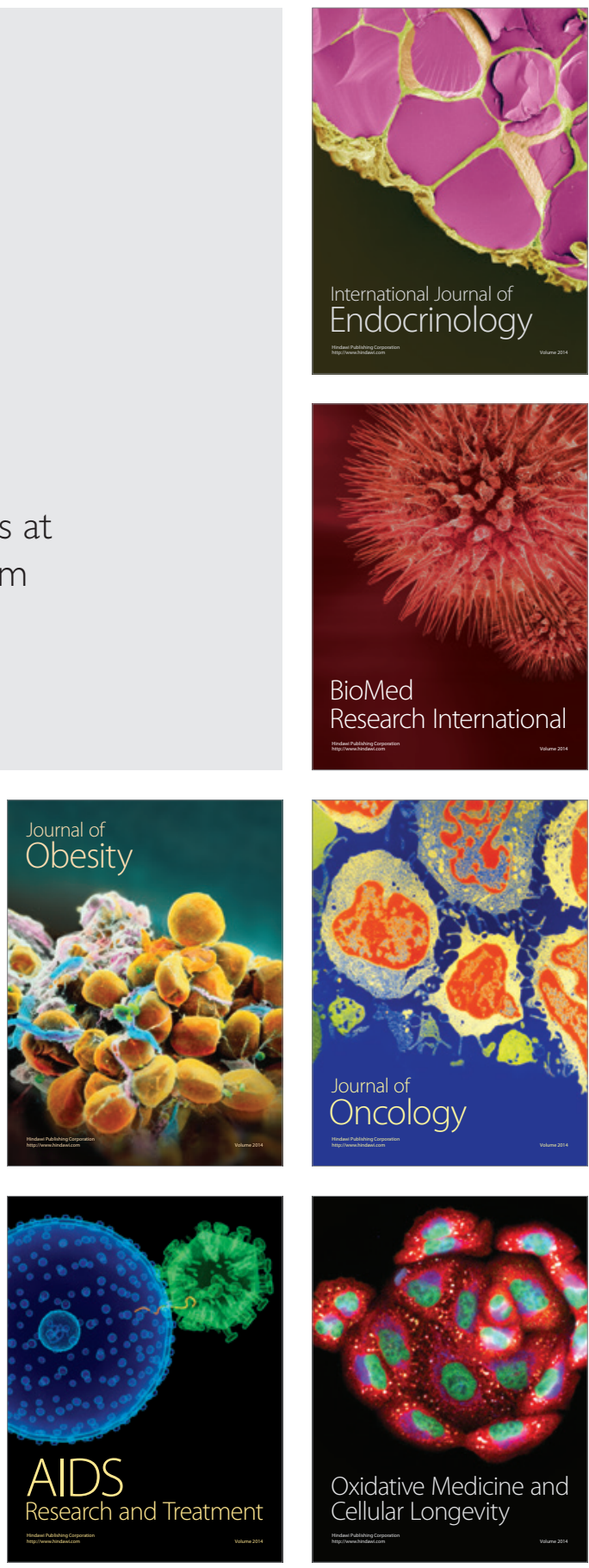